\title{
Distribution system operation supported by contextual energy resource management based on intelligent SCADA
}

\author{
Zita Vale*, Hugo Morais, Pedro Faria, Carlos Ramos
}

\begin{abstract}
Future distribution systems will have to deal with an intensive penetration of distributed energy resources ensuring reliable and secure operation according to the smart grid paradigm. SCADA (Super- visory Control and Data Acquisition) is an essential infrastructure for this evolution. This paper proposes a new conceptual design of an intelligent SCADA with a decentralized, flexible, and intelligent approach, adaptive to the context (context awareness).

This SCADA model is used to support the energy resource management undertaken by a distribution network operator (DNO). Resource management considers all the involved costs, power flows, and electricity prices, allowing the use of network reconfiguration and load curtailment. Locational Marginal Prices (LMP) are evaluated and used in specific situations to apply Demand Response (DR) programs on a global or a local basis.

The paper includes a case study using a 114 bus distribution network and load demand based on real data.
\end{abstract}

Keywords:

Distributed energy resources, Distributed generation, Distribution systems, SCADA, Smart grid

\section{Introduction}

Power systems are essential for the modern society due to the importance that electricity assumes for the daily life and for the countries' economy and security. Currently, power systems already accommodate a substantial penetration of Distributed Generation (DG) and operate in competitive environments. In the future, power systems will have to deal with large-scale integration of DG and other Distributed Energy Resources (DER), such as storage and Electric Vehicles (EVs), and must ensure a reliable, secure, and flexible operation according to the paradigm of smart grids [1e5]. Other important resource that is gaining increasing relevance in power systems and electricity markets is Demand Response (DR) which includes all intentional electricity consumption pattern modifications by end-use customers that are intended to modify the timing, level of instantaneous demand, or total electricity consumption in response to changes in the price of electricity over time [6e8].

In order to evolve to successful practical implementation of the smart grid paradigm, distribution networks require new protection, control and operation philosophy to cope with these challenges [9].
Future power systems will operate using the decentralized paradigm and also a re-aggregation philosophy, led by strategic coalitions [10].

Supervisory Control and Data Acquisition (SCADA) system are a vital infrastructure for this evolution [11,12]. SCADA systems, traditionally used in larger and higher levels of power systems, to transmit measurements, status information, and control signals to and from Remote Terminal Units (RTUs) must be changed to play their expected functions at the core of the concept of smart grid $e$ the grid of the future [13]. Future SCADA must be designed to support multi-level decentralized decisions and actions. These are the result of smart and strategic behavior of the involved agents $[10,14]$, including power suppliers, networks and consumers and also of smart components and control. A significant number of works is contributing to the diversity of new characteristics required for power system future SCADA systems and also evidencing challenges that they must face.

The authors in [15] propose a model to detect situations of false data injections attacks in power systems and to set protection strategies. These attacks increase with the growing dimension of SCADA systems in smart grids, including high dissemination of wireless networks. The use of Internet and other wireless networks is envisaged for demand-side management, implementing residential load management in the context of smart homes [16e18].

In this paper a conceptual design of an intelligent SCADA, with a more decentralized, flexible, and intelligent approach, adaptive to 


$$
\begin{aligned}
& \text { Period (t) } \\
& \begin{array}{llllllllllll}
1 & 2 & 3 & 4 & 5 & 6 & 7 & 8 & 9 & 10 & 11 & 12
\end{array}
\end{aligned}
$$

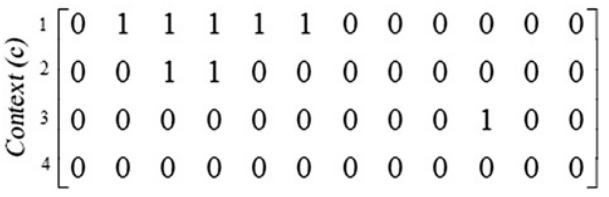

Fig. 1. Example of context occurrence.

the context (context awareness) is used to support energy resource management in the context of future distribution systems [9]. Once a situation is characterized, data and control options available to each entity are redefined according to this context, taking into account operation normative and a priori established contracts. Intelligent SCADA gives Distribution Network Operator (DNO) access to relevant data concerning third-party owned DG, in case of a priori contracted situations (e.g. to manage incident situations, to undertake service restoration or to manage voltage profile).

According to SCADA permissions, DNOs should effectively and efficiently manage the available DER $[2,3,19,20]$. This paper presents an optimization tool able to consider the available resources (generation resources including DG, storage, demand response, and distribution network) and demand requirements in order to minimize operation costs. The presented tool considers all the relevant costs including losses costs, considering AC power flow, providing, as a result, the energy resource scheduling along a multi-period time horizon defined by the user. Network reconfiguration and load curtailment are allowed, according to a priori defined conditions.

Locational Marginal Prices (LMP) are obtained as a result of the optimization process. Based on LMP values, Demand Response (DR) programs can be activated on a network or on a local basis. This feature allows to effectively address high demand and high generation cost situations for which a relatively small decrease in demand may have a significant impact lowering operation costs.

The paper will be organized as follows: after the initial introductory section, Section 2 will deal with the proposed model and present its mathematical model. The application of the proposed model is illustrated with the energy resource management undertaken by a DNO. Section 3 will present a case study using a 114 bus distribution network, adapted from the 123 buses IEEE distribution network. Section 4 presents the most important conclusions of the presented work.

\section{Mathematical model and problem formulation}

This section presents the proposed methodology in three subsections. Sub-section A provides the reader with an explanation of the basic concepts used to build the model. Sub-section B presents the mathematical representation of the proposed model. Finally, sub-section $\mathrm{C}$ presents the mathematical formulation of the optimization problem that aims at the minimization of the DNO operation costs, using the model presented in the two first subsections.

\subsection{Basic concepts}

The efficient operation of distribution systems, taking into account the information concerning the operational network context and the contractual agreements between the involved players, requires the use of new intelligent and flexible SCADA models. This sub-section introduces the basic concepts that allow establishing the basis of the proposed SCADA model.

For better understanding of the model, let us define some terms used in the present work:

- Additional resource e a resource $(r)$ or a partial capacity of a specific resource that is contracted to be used in a determined context $(c)$;

- Context e a priori defined network context that can be characterized by the status of network components (e.g. branches, breakers and switches) and/or by other relevant information such as the demand level, the storage (e.g. storage units, hydro plant reservoirs) status, and the market price. A determined contextcan occur at any period $(t)$ and can last for several periods;

- State e set of contexts occurring in a determined period $(t)$.

For illustration of these concepts, an example considering four contexts is presented below. Fig. 1 presents an example of the occurrence of the four hypothetic contexts along a twelve-period scenario. The information concerning the occurrence of each context $(c)$ is therefore organized in a two-dimension matrix. Looking at a column of this matrix, which contains the information related to a period $(t)$, it is possible to identify the state of the network in thatperiod.

In this example, ten additional resources can be used by the DNO, regarding the permissions established according to the existing contracts in the four considered contexts, as seen in Fig. 2. Looking at a row of this matrix, which corresponds to a certain specific context, it is possible to identify which additional resources are available for the DNO use in that context.

\subsection{Mathematical representation of the model}

This sub-section presents the mathematical formulation of the proposed SCADA model.

The occurrence of contexts over time, as defined in the previous sub-section, can be represented by a matrix formulated as in equation (1) which is hereinafter referred as Ct_matrix. Each line of this matrix corresponds to a specific context $c$. The element $c t_{c, t}$ is equal to " 1 " if the context $c$ occurs in period $t$, and equal to " 0 " otherwise. $N$ and $T$ are the cardinality of contexts and periods, respectively.

$$
C_{-} \text {matrix }=\left[\begin{array}{ccccccc}
c t_{1,1} & c t_{1,2} & \cdots & c t_{1, t} & \cdots & c t_{1, T-1} & c t_{1, T} \\
c t_{2,1} & c t_{2,2} & \cdots & c t_{2, I} & \cdots & c t_{2, T-1} & c t_{2, T} \\
\vdots & \vdots & \ddots & \vdots & \ddots & \vdots & \vdots \\
c t_{c, 1} & c t_{c, 2} & \cdots & c t_{c, t} & \cdots & c t_{c, T-1} & c_{c, T} \\
\vdots & \vdots & \ddots & \vdots & \ddots & \vdots & \vdots \\
c t_{N-1,1} & c t_{N-1,2} & \cdots & c t_{N-1,1} & \cdots & c t_{N-1, T-1} & c t_{N-1, T} \\
c t_{N, 1} & c t_{N, 2} & \cdots & c t_{N, I} & \cdots & c t_{N, T-1} & c t_{N, T}
\end{array}\right]
$$




$$
\begin{aligned}
& \text { Additional resource(r) } \\
& \begin{array}{llllllllll}
1 & 2 & 3 & 4 & 5 & 6 & 7 & 8 & 9 & 10
\end{array}
\end{aligned}
$$

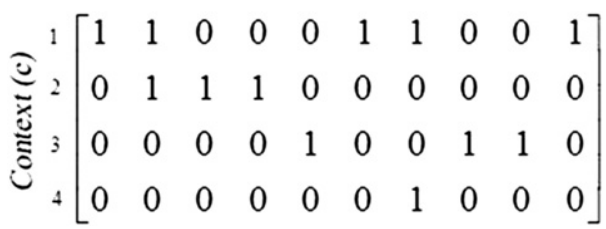

Fig. 2. Example of additional resources availability in each context.

The considered contexts are a priori defined contexts that are of particular relevance to the network operation. DNOs should define the set of relevant contexts and negotiate with other players the contractual conditions to use some of their resources that are useful for each considered context. Consequently, the contractually allowed third party resource use is contextual.

$R c \_a d d i t i o n a l \_m a t r i x$, as seen in equation (2), includes the additional (third party) resources that can be used in each considered context $c$. This matrix corresponds to the one presented in Fig. 2 for the example in the previous sub-section. The element $r_{c, r}$ is equal to " 1 " if the additional resource $r$ is contracted to be used in context $c$, and " 0 " otherwise. $N$ and $R_{-} a d$ are the cardinality of contexts and additional resources, respectively.

$$
\text { Rc_additional_matrix }=\left[\begin{array}{ccccccc}
r_{1,1} & r_{1,2} & \cdots & r_{1, x} & \cdots & r_{1, R \_a d-1} & r_{1, R \_a d} \\
r_{2,1} & r_{2,2} & \cdots & r_{2, x} & \cdots & r_{2, R \_a d-1} & r_{2, R \_a d} \\
\vdots & \vdots & \ddots & \vdots & \ddots & \vdots & \vdots \\
r_{c, 1} & r_{c, 2} & \cdots & r_{c, x} & \cdots & r_{c, R \_d-1} & r_{c, R \_a d} \\
\vdots & \vdots & \ddots & \vdots & \ddots & \vdots & \vdots \\
r_{N-1,1} & r_{N-1,2} & \cdots & r_{N-1, x} & \cdots & r_{N-1, R \_a d-1} & r_{N-1, R \_a d} \\
r_{N, 1} & r_{N, 2} & \cdots & r_{N, x} & \cdots & r_{N, R \_d-1} & r_{N, R-a d}
\end{array}\right]
$$

$R \_p o w e r \_m a t r i x$, as seen in equation (3), registers the information considering the maximum available power for each resource (matrix row) in each period (matrix column, from $t 1 / 41$ to $T$ ).

$$
\text { R_power_matrix }=\left[\begin{array}{ccccccc}
P_{1,1} & P_{1,2} & \cdots & P_{1, x} & \cdots & P_{1, R-1} & P_{1, R} \\
P_{2,1} & P_{2,2} & \cdots & P_{2, x} & \cdots & P_{2, R-1} & P_{2, R} \\
\vdots & \vdots & \ddots & \vdots & \ddots & \vdots & \vdots \\
P_{t, 1} & P_{t, 2} & \cdots & P_{t, x} & \cdots & P_{t, R-1} & P_{t, R} \\
\vdots & \vdots & \ddots & \vdots & \ddots & \vdots & \vdots \\
P_{T-1,1} & P_{T-1,2} & \cdots & P_{T-1, x} & \cdots & P_{T-1, R-1} & P_{T-1, R} \\
P_{T, 1} & P_{T, 2} & \cdots & P_{T, x} & \cdots & P_{T, R-1} & P_{T, R}
\end{array}\right]
$$

$R \_$cost_matrix, as seen in equation (4), registers the information considering the cost of each resource (matrix row) in each period (matrix column, from $t^{1 / 4} 1$ to $T$ ).

•
The unavailable resources in a determined period should also be represented in the model. This information is coded in the matrix $R \_u n a v a i l a b l e$, as seen in equation (5), a matrix with two rows and $R$ columns, as many as all the considered resources (i.e. the ones owned by the DNO and the third party resources, for which the use, under determined context(s), is contracted).

The first row pertains to the unavailability starting period $(t r)$ of the situation, and the second one to the expected duration $(d r)$ of the unavailability, for each resource $(r)$.

$$
\text { R_unavailable }=\left[\begin{array}{ccccccc}
t_{1} & t_{2} & \cdots & t_{r} & \cdots & t_{R-1} & t_{R} \\
d_{1} & d_{2} & \cdots & d_{r} & \cdots & d_{R-1} & d_{R}
\end{array}\right]
$$

This matrix is permanently updated using the information about all the considered resources. For a specific period $t, t r$ is:

- $t r: \mathrm{s} t$, if the resource $r$ is already unavailable;

- $\operatorname{tr} 2^{\prime} t$, if resource $r$ is expected to be unavailable, starting in period $t r$

- zero if there is no information concerning a confirmed or expected unavailability situation for resource $r$.

A third row can be considered, registering a confidence measure concerning the information registered in rows 1 and 2, i.e., the third line would include the probability of the information about resource $r$ being true. This probability is especially important for

resources for which $t r$ is set to zero, although there is no reliable information about the resource state.

The following sets are also used in the proposed model:

(3)

$R \_$additional e set of all additional resources;

- $R \_$additional $c$ e Additional resources available in context $c$, i.e. the additional resources available in context $c$ as

$$
R_{-} \text {cost_matrix }=\left[\begin{array}{ccccccc}
c_{1,1} & c_{1,2} & \cdots & c_{1, t} & \cdots & c_{1, T-1} & c_{1, T} \\
c_{2,1} & c_{2,2} & \cdots & c_{2, t} & \cdots & c_{2, T-1} & c_{2, T} \\
\vdots & \vdots & \ddots & \vdots & \ddots & \vdots & \vdots \\
c_{x, 1} & c_{x, 2} & \cdots & c_{x, t} & \cdots & c_{x, T-1} & c_{x, T} \\
\vdots & \vdots & \ddots & \vdots & \ddots & \vdots & \vdots \\
c_{R-1,1} & c_{R-1,2} & \cdots & c_{R-1, t} & \cdots & c_{R-1, T-1} & c_{R-1, T} \\
c_{R, 1} & c_{R, 2} & \cdots & c_{R, t} & \cdots & c_{R, T-1} & c_{R, T}
\end{array}\right]
$$


indicated in C_matrix (only the resources which corresponding elements assume the value "1" in row $c$ are considered);

- $R \_$additional $l_{t}$ e Additional resources usable in period $t$;

- $R \_$base e Base resources (the ones owned by the DNO) that can be used in any context or period;

- $R \_$global e set of all the resources including the base resources and the additional resources;

- $R \_g_{\text {global }}$ e set of resources that can be used in period $t$, according to the state of the system in that period;

- $R_{-}$opt e set of resources available for operation in period $t$;

- $R_{t}$ e usable resources in period $t$

- $R \_$unavailable $e_{t}$ e set of resources unavailable in period $t$ for which the following cardinality values are relevant:

- $N \_D G$ e cardinality of base resources of type DG (Distributed Generation);

- $N_{-} L C_{-} 1$ e cardinality of base resources of type $L C_{-} 1$;

- $N \_$storage e cardinality of base resources of type storage;

- $R$ e cardinality of $R \_$global.

In what concerns the temporal variables, $t$ is used as a single period index.

R_base can be obtained as the gathering of the base resources sets concerning the considered resource types, as expressed in equation (6). For this formulation, we are considering the following types of base resources: distributed generation (DG) units, storage units and one load curtailment program (LC_1). External energy suppliers are also considered as base resources.

$$
R_{\_} \text {additional }_{t}=\bigcup_{c=1}^{N}\left(R \_ \text {additional }{ }_{c} \cap C_{c, t}\right)
$$

$R \_$global $_{t}$ can be defined as the relative complement of $R_{-}$unavailable $t_{t}$ in $R_{-}$global (also known as the set-theoretic difference of $R \_g l o b a l$ and $R \_$unavailable $t$ ), as expressed in equation (11).

$$
R_{\_} \text {global }_{t}=R_{\llcorner} \text {global }-R_{\_} \text {unavailable } t
$$

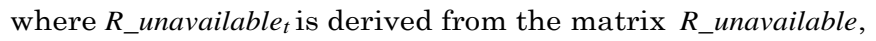
updated for period $t$.

Using the above defined sets, the set of the usable resources in period $t, R_{t}$, can be expressed as in equation (12).

$$
R_{t}=R \_ \text {base } \cup \text { R_additional } t
$$

Out of the resources in set $R_{t}$, only the ones that are available $\left(R \_o p_{t}\right)$ in the considered period $(t)$ can be used, as expressed in equation (13).

$$
\text { - } \quad R_{-} o p_{t}=R_{t} \cap R_{-} \text {global }_{t}
$$

\subsection{Optimization problem}

The problem employed in this paper to illustrate the use of the proposed model concerns the minimization of the operation costs of the DNO, as expressed in equation (14).

Additionally to the variables already defined in sub-section B, the following variables are also used in the mathematical formulation of this problem:
$R \_$additional can be obtained as the gathering of the additional resources sets concerning the considered contexts, as expressed in equation (7).

$$
\text { R_additional }=\bigcup_{c=1}^{N} R_{c} \_ \text {additional } l_{c}
$$

We are considering the following types of additional resources: distributed generation (DG) units, storage units and additionalload curtailment programs. Additional external energy suppliers are also considered.

$R \_$global can be obtained as the gathering of $R \_$base and $R \_$additional, as expressed in equation (8).

$$
R \_ \text {global }=R \_ \text {_base } U R \_a d d i t i o n a l
$$

The use of the proposed model requires the definition of sets with similar meaning as the ones referred above but dynamically updated for each period $t$.

Let us consider an auxiliary set $C_{c, t}$ as seen in equation (9), which equals $R \_$global or the empty set, respectively if the context $c$ occurs or not in period $t$.

$$
C_{c, t}=\left\{\begin{array}{l}
\varnothing \text { if } c_{c, t}=0 \\
R_{-g l o b a l} \text { if } c_{c, t}=1
\end{array}\right.
$$

where $c_{c, t}$ are elements of $C_{-}$matrix.

$R \_$additional $l_{t}$ can be defined as the gathering of this auxiliary set with the set of additional resources that can be used in each context $c, R \_$additional $c$, as expressed in equation (10). $\mathrm{q}_{b, t}$ e Voltage angle in bus $b$ in period $t$

- $\mathrm{q}_{k, t}$ e Voltage angle in bus $k$ in period $t$;

- $q_{\text {min }}^{\max }$ e Maximum voltage angle in bus $b$;

- $\mathrm{q}_{b}$ e Minimum voltage angle in bus $b$;

- $\mathrm{h}_{c(r 1)}$ e Storage $r 1$ charge efficiency;

- $\mathrm{h}_{d(r 1)}$ e Storage $r 1$ discharge efficiency;

- D $t$ e Time period interval;

- $c_{r, t}$ e Cost of resource $r$ in period $t$

- $c_{E G E(r, t)}$ e Cost of excess active power generated by resource $r$ in period $t$

- $c_{N S E(r, t)}$ e Cost of active power not supplied to the load $L$ in period $t$;

- $y_{b k}$ e Admittance of the line that connects bus $b$ and $k$;

- $y_{\text {Shunt_b }}$ e Shunt admittance of bus $b$;

- $E_{\text {Capacity }(r 1)}$ e Storage $r 1$ energy limit;

- $E_{\text {Stored }(r 1, t)}$ e Active energy stored in storage unit $r 1$ in period $t$;

- $E_{S t o r e d}(r 1, t-1)$ e Active energy stored in storage unit $r 1$ in period $t-1$

- $N_{b}$ e Total number of bus;

- $N_{L}$ e Total number of loads;

- $P_{r, t}$ eScheduledactivepowergeneration of resource $r$ in period $t$;

- $P_{b r, t}$ e Scheduled active power generation of resource $r$ in bus $b$, in period $t$

- $P_{\text {charge }(r 1, t)}$ e Power charge in storage unit $r 1$ in period $t$;

- $P_{b}$ hargeLimit $(r 1, t)$ e Powerchargelimitin storage unit $r 1$ in period $t$;

- $P$ charge $(r 1, t)$ e Power charge in storage unit $r 1$ in bus $b$, in period $t$;

- $P_{\text {limit }(r, t)}$ e Maximum active power generation of resource $r$ in period $t$

- $P_{E G E(r, t)}$ e Excessactivepowergenerated byresourcerinperiod $t$;

- $P_{E G E(r, t)}^{b}$ e Excess active power generated by resource $r$ in bus $b$, in period $t$; 


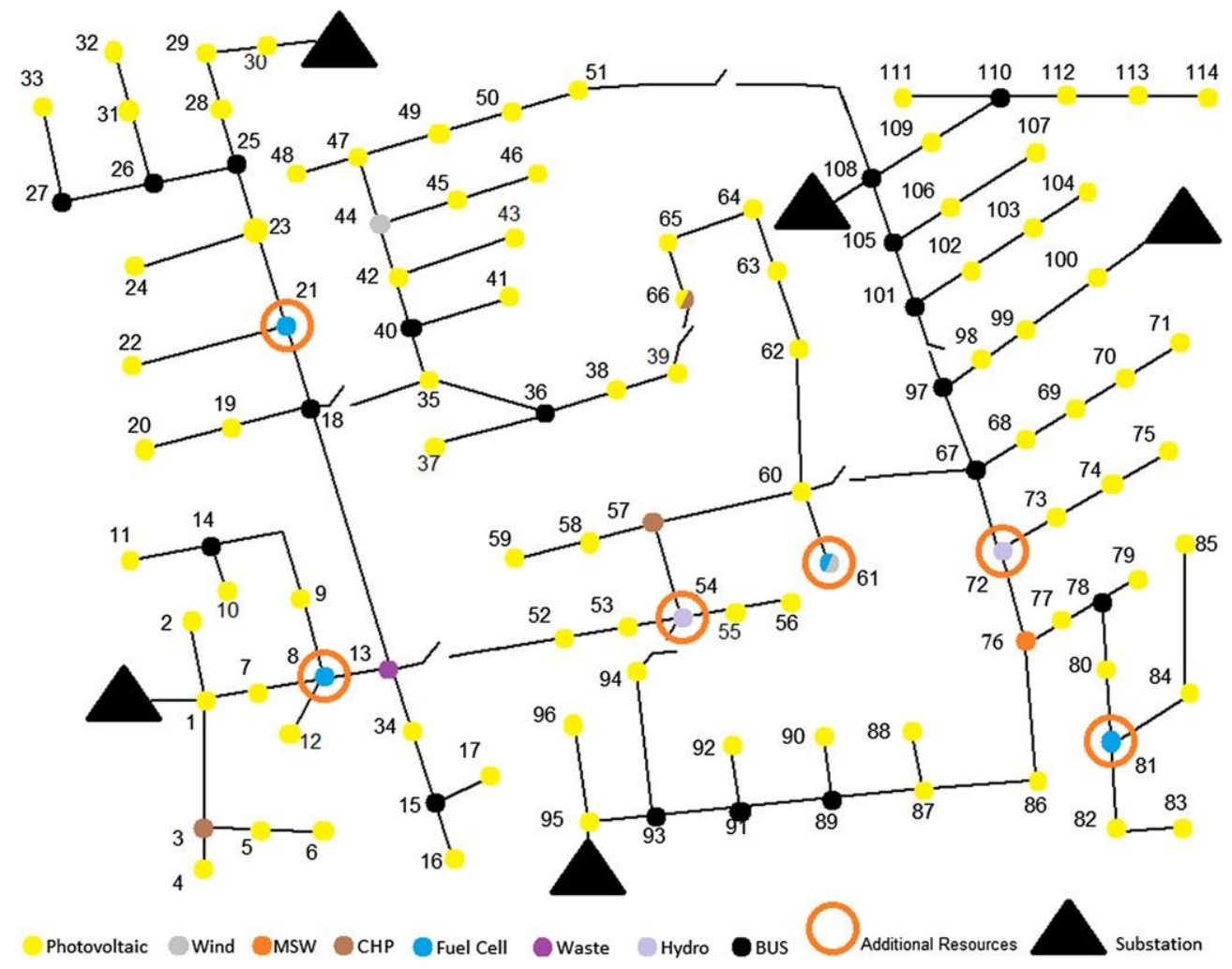

Fig. 3. Case study 114 bus distribution network.

- $P_{\text {Load }(r, t)}^{b}$ e Active power demand of load $L$ in bus $b$, in period $t$;

- $P_{N S E(L, t)}$ e Active power not supplied to load $L$ in period $t$;

- $P^{b}{ }_{N S E(L, t)}$ e Active power not supplied to $\operatorname{load} L$ in bus $b$, in period ;

- $Q_{r, t}$ e Reactive power generation of resource $r$ in period $t$;

- $Q_{b r, t}$ e Reactive power generation of resource $r$ in bus $b$, in period $t$

- $Q_{\text {limit }(r, t)}$ e Maximum reactive power generation of resource $r$ in period $t$

- $Q^{b}{ }_{\text {Load }(r, t)}$ e Reactive power demand of load $L$ in bus $b$, in period $t$

- $Q^{b}{ }_{N S E(L, t)}$ e Reactive power not supplied to the load $L$ in bus $b$, in period $t$;
- $R_{m+t} s t_{t}$ e Storage units resources in period $t$;

- $S_{b k}$ e Maximum apparent power flow in the line that connects

- bus $b$ and $k$;

- $V_{b}^{\max }$ e Maximum voltage magnitude in bus $b$;

- $V_{b}^{\min }$ e Minimum voltage magnitude in bus $b$

- $X_{r 1, t}$ e Binary variable of storage unit $r 1$ in period $t$, related to power discharge;

- $Y_{r 1, t}$ e Binary variable of storage unit $r 1$ in period $t$, related to power charge.

The presented formulation is generic for a specified time period (from period $t^{1 / 4} 1$ to $t^{1 / 4} T$ ).

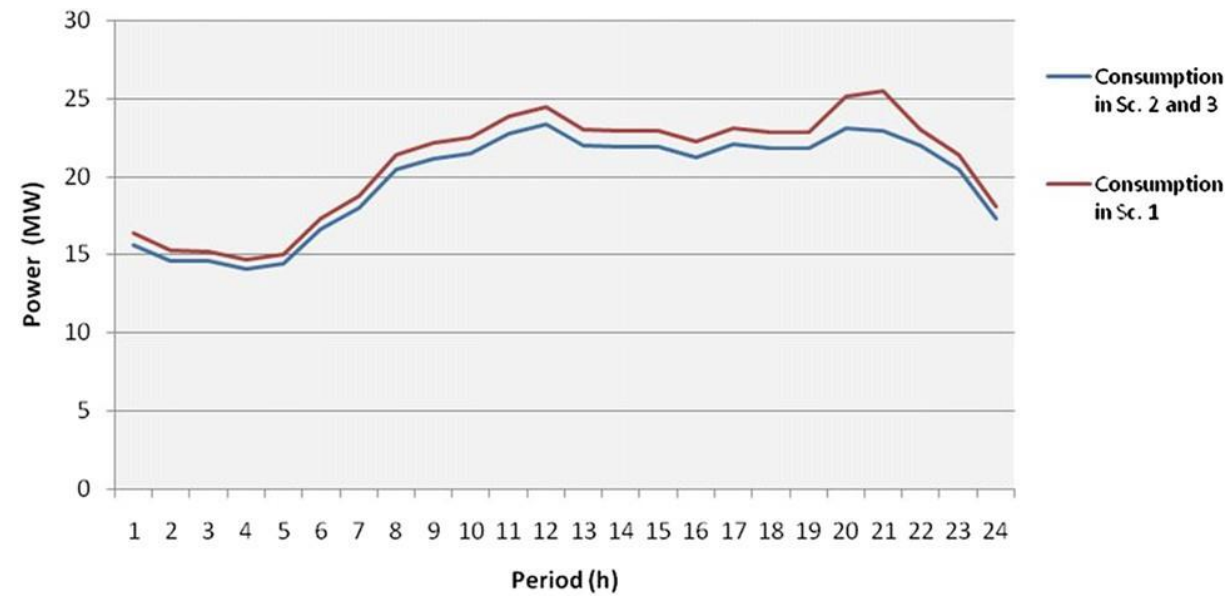

Fig. 4. Demand for the 24-h simulation. 


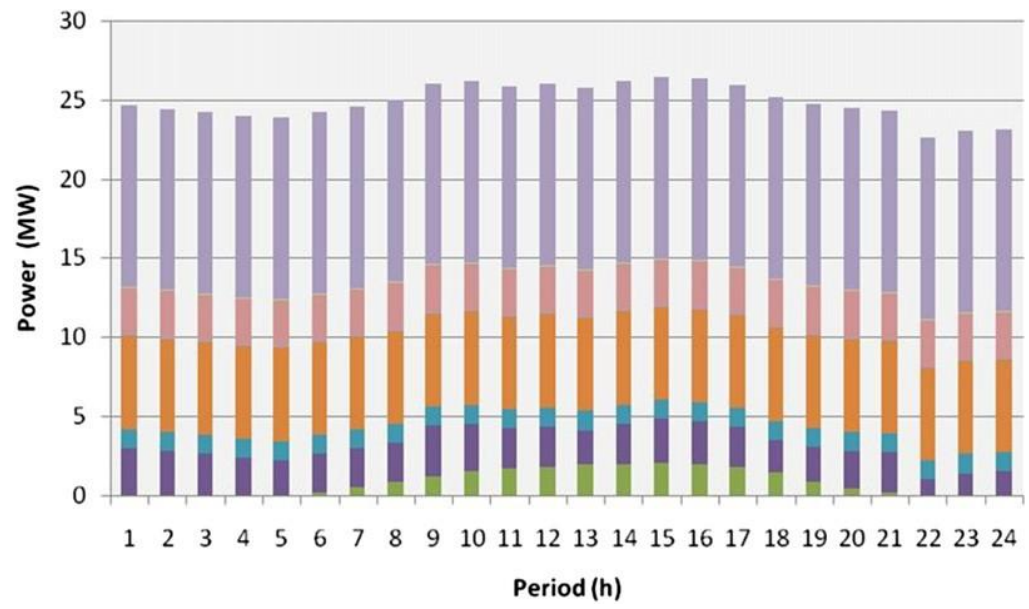

Euppliers

\#ydro

E Biomass

a Fuelcell

$=\mathrm{CHP}$

MSW

a Wind

Ehotovoltaic

Fig. 5. Base resources for the first day simulation.

$$
f=\sum_{t=1}^{T}\left(\sum_{r=1, r=R_{-} a p_{t}}^{R}\left(P_{r, t} \times c_{r, t}+P_{E C E(r, t)} \times c_{E C E E(r, t)}\right)+\sum_{L=1}^{N_{2}}\left(P_{\operatorname{NSE}(L, t)} \times c_{N S E(L, t)}\right)\right)
$$

This problem is a Mixed-Integer Non Linear Programming (MINLP) problem. All the involved costs are represented by sournear cost function sievond the rempneration of toeneration

discharge, and demand response are also included ( $c_{r, t}$ in equation (14)). The penalties include non-supplied energy $\left(c_{N S E(r, t)}\right)$ and the excess generated energy $\left(c_{E G E(r, t)}\right)$ costs.

The efficient management of the available energy resources requires a multi-period optimization. The minimization of this objective function is subjected to the following constraints:

- The network reactive power balance in each bus $b$ and each period $t$

- The network active power balance in each bus $b$ and each period $t$

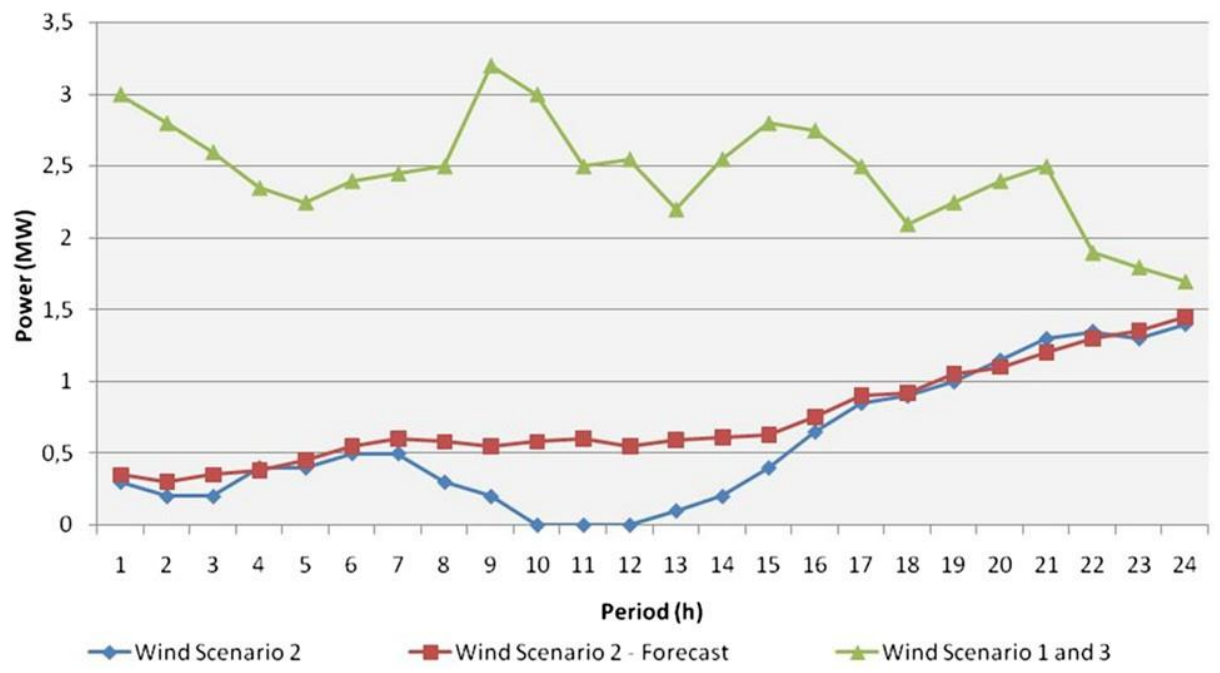

Fig. 6. Wind generation in the considered scenarios. 
Table 1

Case study contexts.

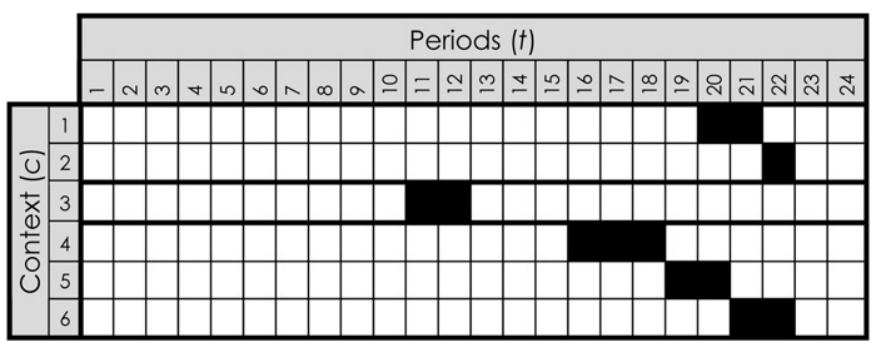

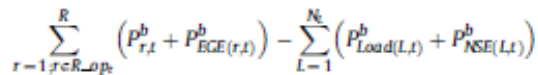

$-\sum_{r_{1}=1 \text {; re R_st, }}^{R}\left(P_{\text {charge }\left(r_{1}, t\right)}^{b}\right)=\sum_{k=1 ; k+b}^{N_{b}} V_{b, t} \times V_{k, t}$

$\times\left(G_{b k} \times \cos \left(\theta_{b, t}-\theta_{k, t}\right)+B_{b k} \times \sin \left(\theta_{b, t}-\theta_{k, t}\right)\right)$

- Bus voltage magnitude in equation (17) and angle limits in equation (18);

$V_{b}^{\min } \leq V_{b, t} \leq V_{b}^{\max }$

$\forall t \in\{1, \ldots, T\} ; \forall b$ and $\forall k \in\left\{1, \ldots, N_{b}\right\}$

$\theta_{b}^{\min } \leq \theta_{b, t} \leq \theta_{b}^{\max }$

$\forall t \in\{1, \ldots, T\} ; \forall b$ and $\forall k \in\left\{1, \ldots, N_{b}\right\}$

- Line thermal limits;

$$
\begin{aligned}
& \text { ( } \\
& \left|V_{b, t} \times\left(\left[\left(V_{b, t}-V_{k, t}\right) y_{b k}\right]^{*}+\left[V_{b, t} \times \frac{1}{2} y_{\text {Shunt }, b}\right]\right)\right| \leq S_{b k}^{\max } \\
& \forall t \in\{1, \ldots, T\} ; \forall b \text { and } \forall k \in\left\{1, \ldots, N_{b}\right\}
\end{aligned}
$$

- Maximum active in equation (20) and reactive in equation (21) resources limit in each period $t$;

$$
\begin{aligned}
& P_{r, t}+P_{E C E}(r, t) \leq P_{\text {limit }(r, t)} \\
& \forall t \in\{1, \ldots, T\} ; \forall r \in\left\{1, \ldots, R_{\_} o p_{t}\right\} \\
& \left.\left.Q_{r, t} \leq Q_{\text {limit }}\right\} r, t\right) \\
& \forall t \in\{1, \ldots, T\} ; \forall r \in\left\{1, \ldots, R_{\_} o p_{t}\right\}
\end{aligned}
$$

- Storage units resources needs specific çonstraints:

- Storage charge and discharge are not simultaneous;

$$
X_{r_{1}, t}+Y_{r_{1}, t} \leq 1
$$

$\forall t \in\{1, \ldots, T\} ; \quad \forall r_{1} \in\left\{1, \ldots, R \_t_{t}\right\}$

$X_{\left(r_{1}, t\right)}$ and $Y_{\left(r_{1} t\right)} \in\{0,1\}$

- Batterybalance for each storage unit:

$E_{\text {Stured }\left(r_{1}, t\right)}=E_{\text {Stared }\left(r_{1}, t-1\right)}+\eta_{c\left(r_{1}\right)} \times P_{C h a r g e\left(r_{1}, t\right)} \times \Delta t-\frac{1}{\eta_{d\left(r_{1}\right)}} \times P_{\left(r_{1}, t\right)} \times \Delta t$

$\forall t \in\{1, \ldots, T\} ; \quad \forall r_{1} \in\left\{1, \ldots, r \in R \_s t_{t}\right\} ; \quad \Delta t=1 ;$
Table 2

Additional resources in each context.

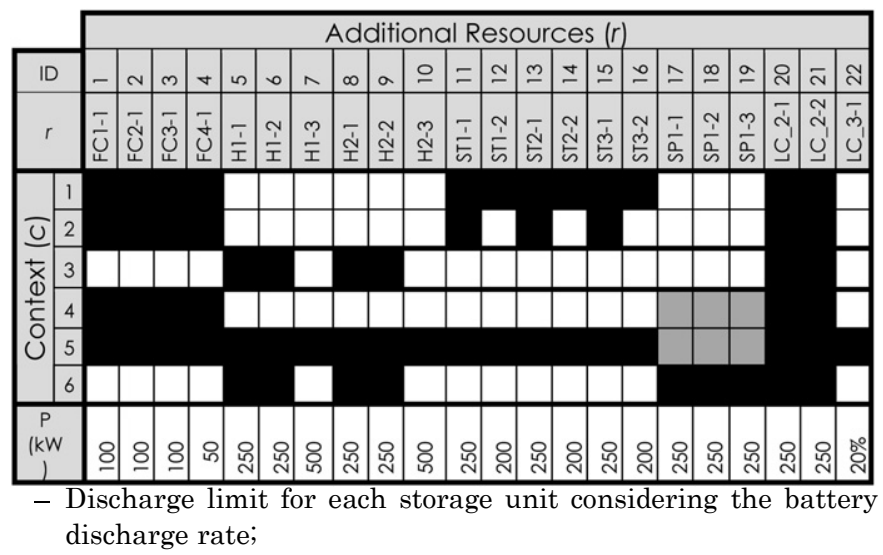

$$
\begin{aligned}
& P_{\left(r_{1}, t\right)} \leq P_{\text {limit }\left(r_{1}, t\right)} \times X_{\left(r_{1}, t\right)} \\
& \forall t \in\{1, \ldots, T\} ; \quad \forall r_{1} \in\left\{1, \ldots, r \in R \_s t_{t}\right\} \quad X_{\left(r_{1}, t\right)} \in\{0,1\}
\end{aligned}
$$

- Charge limit for each storage unit considering the battery charge rate;

$$
\begin{aligned}
& P_{\text {charge }}\left(r_{1}, t\right) \leq P_{\text {chargedimit }}\left(r_{1}, t\right) \\
& \forall t \in\left\{Y_{\left(r_{1}, t\right)}\right. \\
& \forall t, \ldots, T\} ; \quad \forall r_{1} \in\left\{1, \ldots, r \in R \_s t_{t}\right\} ; \quad Y_{\left(r_{1}, t\right)} \in\{0,1\}
\end{aligned}
$$

- Storage unit discharge limit considering the storage balance;

$$
\begin{aligned}
& \frac{1}{\eta_{d\left(r_{1}\right)}} \times P_{r_{1}, t} \times \Delta t \leq E_{S \operatorname{sed}\left(r_{1}, t-1\right)} \\
& \forall t \in\{1, \ldots, T\} ; \quad \forall r_{1} \in\left\{1, \ldots, r \in R_{-} s t_{t}\right\} ; \quad \Delta t=1 ;
\end{aligned}
$$

Storage charge limit considering the capacity and previous charge status;

$$
\begin{aligned}
& \eta_{c(V)} \times P_{\text {Oharge }\left(r_{1}, t\right)} \times \Delta t \leq E_{\text {Capaciy }\left(r_{1}\right)}-E_{\text {Swred }\left(r_{1}, t-1\right)} \\
& \forall t \in\{1, \ldots, T\} ; \quad \forall r_{1} \in\left\{1, \ldots, r \in R_{-} s t_{t}\right\} ; \quad \Delta t=1 ;
\end{aligned}
$$

- Storage capacity limit;

$$
\begin{aligned}
& \left.E_{\text {Stared }\left(r_{1}, t\right)} \leq E_{\text {Capacity }\left(r_{1}\right)}, T, \ldots, r \in R \_s t_{t}\right\} \\
& \forall t \in\left\{1, \ldots, \quad \forall r_{1} \in\{1, \ldots,\right.
\end{aligned}
$$

The above formulation has been implemented in GAMS [21].

3. Case study

The case study presented in this section is based on a 114 bus distribution network, adapted from the 123 buses IEEE 
Table 3

Additional resources costs in each context (m.u.).

\begin{tabular}{|c|c|c|c|c|c|c|c|c|}
\hline & & & \multicolumn{6}{|c|}{ Context (c) } \\
\hline & ID & $r$ & 1 & 2 & 3 & 4 & 5 & 6 \\
\hline \multirow{22}{*}{ 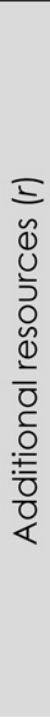 } & 1 & $\mathrm{FCl}_{1} 1$ & 0.17 & 0.17 & 0.17 & 0.17 & 0.17 & 0.17 \\
\hline & 2 & FC2_1 & 0.17 & 0.17 & 0.17 & 0.17 & 0.17 & 0.17 \\
\hline & 3 & FC3_1 & 0.17 & 0.17 & 0.17 & 0.17 & 0.17 & 0.17 \\
\hline & 4 & FC4_1 & 0.45 & 0.45 & 0.45 & 0.45 & 0.45 & 0.45 \\
\hline & 5 & $\mathrm{HI}$ l & 0.20 & 0.2 & 0.06 & 0.06 & 0.06 & 0.06 \\
\hline & 6 & $\mathrm{HI} 2$ & 0.22 & 0.22 & 0.08 & 0.08 & 0.08 & 0.08 \\
\hline & 7 & $\mathrm{HI} 3$ & 0.25 & 0.25 & 0.09 & 0.09 & 0.09 & 0.09 \\
\hline & 8 & $\mathrm{H} 2 \_\mathrm{I}$ & 0.20 & 0.2 & 0.06 & 0.06 & 0.06 & 0.06 \\
\hline & 9 & $\mathrm{H} 2 \_2$ & 0.22 & 0.22 & 0.08 & 0.08 & 0.08 & 0.08 \\
\hline & 10 & $\mathrm{H} 2 \_3$ & 0.25 & 0.25 & 0.09 & 0.09 & 0.09 & 0.09 \\
\hline & 11 & STI_l & 0.14 & 0.14 & 0.14 & 0.14 & 0.14 & 0.14 \\
\hline & 12 & STI_2 & 0.16 & 0.16 & 0.16 & 0.16 & 0.16 & 0.16 \\
\hline & 13 & ST2_1 & 0.14 & 0.14 & 0.14 & 0.14 & 0.14 & 0.14 \\
\hline & 14 & ST2_2 & 0.16 & 0.16 & 0.16 & 0.16 & 0.16 & 0.16 \\
\hline & 15 & ST3_1 & 0.14 & 0.14 & 0.14 & 0.14 & 0.14 & 0.14 \\
\hline & 16 & ST3_2 & 0.16 & 0.16 & 0.16 & 0.16 & 0.16 & 0.16 \\
\hline & 17 & SPI_1 & 0.18 & 0.18 & 0.18 & - & - & 0.04 \\
\hline & 18 & SPI_2 & 0.19 & 0.19 & 0.19 & - & - & 0.045 \\
\hline & 19 & SPI_3 & 0.20 & 0.20 & 0.20 & - & - & 0.05 \\
\hline & 20 & DR1_1 & 0.35 & 0.35 & 0.35 & 0.35 & 0.35 & 0.35 \\
\hline & 21 & DR1_2 & 0.40 & 0.40 & 0.40 & 0.40 & 0.40 & 0.40 \\
\hline & 22 & DR2_1 & 0.26 & 0.26 & 0.26 & 0.26 & 0.26 & 0.26 \\
\hline
\end{tabular}

distribution network [22]. The considered network includes 9 storage units, 97 DG units (Fig. 3), and 84 loads which consumer profiles for the considered 24 - $\mathrm{h}$ period are based on real data from the Portuguese distribution utility as presented in [23]. In Fig. 3, the contracted additional resources are identified by the large orange circles.

The proposed methodology is applied to three scenarios, which take place in three distinct days. The first scenario considers abnormal high peak demand on periods 20 and 21 . The second scenario considers the inexistence of wind generation in periods 10,11, 12 and 13 . The third scenario considers an incident state.
The resulting load diagrams for the three scenarios considered in the case study are presented in Fig. 4 . The base resources for each period of the first day are presented in Fig. 5 .

For the two other days, the base resources are considered the same, with the exception of wind generation maximum limit, which is represented in Fig. 6 .

Tables $1 e 3$ represent the information in matrixes Ct_matrix (1), $R c \_a d d i t i o n a l \_m a t r i x(2), R \_p o w e r \_m a t r i x(3)$, and $R \_c o s t \_m a t r i x(4)$. The black squares represent the variables equal to 1 and the white squares the variables equal to 0 . The grey squares represent the resources that are unavailable, according to equation (5). Table 1 presents the contexts that occur in each period of the case study. Scenario 1 corresponds to the successive occurrence of contexts 1 (in periods 20 and 21) and 2 (in period 22). Scenario 2 corresponds to context 3 in periods 11 and 12 . Finally, scenario 3 corresponds to the successive occurrence of contexts 4 (in periods 16,17 and 18), 5 (in periods 19 and 20), and 6 (in periods 21 and 22).

Table 2 includes the information about all the contracted additional resources in each context. In the third row, it can be seen the type of each resource. Each resource type can be identified by the following initials: Fuel cell (FC); Hydro units (H), Storage units (St); Suppliers (Sp); Load Curtailment(LC).

Load curtailment resources correspond to three demand response programs. The first (LC_1) is a Direct Load Control (DLC) that the DNO can use as a base resource. The additional demand response resources (LC_2-1, LC_2-2 and LC_3-1) are included in Table 2. LC_2-1 and LC_2-2 are LMP-triggered demand response programs, inspired on [24], with two successive steps. These programs may be activated when the Locational Marginal Price (LMP) value is above an a priori defined LMP limit. LC_3-1 is a DLC program. Several additional resources can correspond to partial uses of the same physical resource, which are independently contracted. For example, hydro unit 1 is represented by three resources $(r 5, r 6, r 7)$.

The last row of Table 2 presents the maximum contracted power for each resource. In the case of LC_3-1, this value varies throughout the day according to the demand in each period and as a mean value equal to 20 percent.

Table 3 includes the information about the costs of the contracted additional resources in each context.

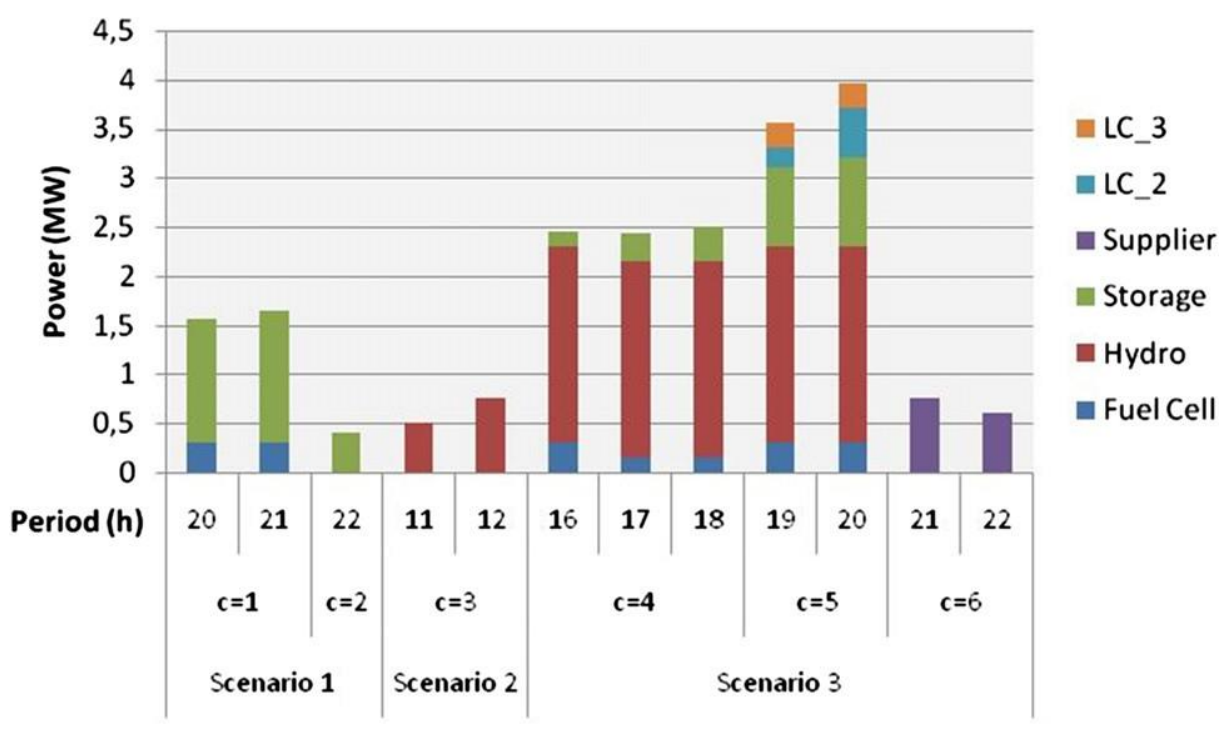

Fig. 7. Additional energy resource used in the three scenarios. 


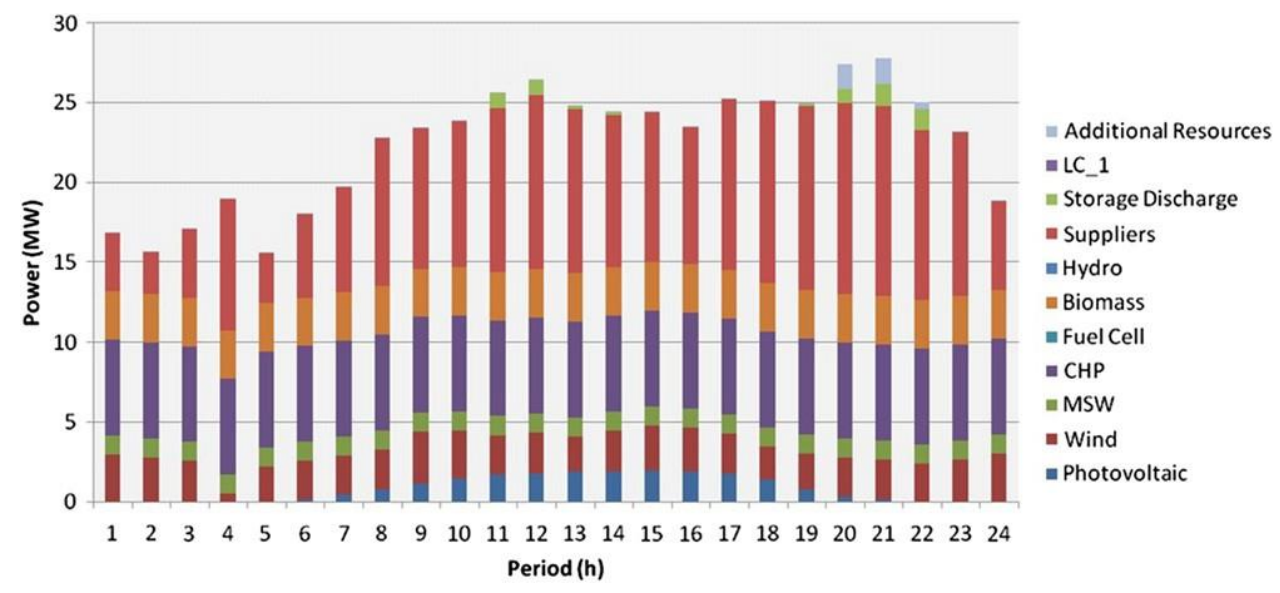

Fig. 8. Energy resource scheduling for scenario 1.

Fig. 7 shows a summary of the additional energy resource scheduling for the three considered scenarios. The obtained scheduling is the result of the minimization of DNO costs, considering all the available resources (base and additional contracted resources) in each period. The next sub-sections present additional details for each scenario.

\subsection{Scenario 1}

This scenario concerns a high demand profile, with peak values over $25 \mathrm{MW}$ in periods 20 and 21. In this context, DNO base resources are not enough to supply all the demand, being necessary to use additional contracted resources. The DNO can activate the contracts of four fuel cells, the first step of three storage units, and LC_2-1 and LC_2-2 for contexts 1 and 2 . In context 1, it can also use the second step of the three storage units.

Normally external suppliers and hydro units are the first choices to supply the demand. However, in the present scenario, the water reserves in the dam reservoirs are lower and the supplier price is higher than the fuel cells and storage units' prices. Although the DNO has some reserve demand response base resources, it prefers to keep this resource as a reserve and uses additional resources to fully supply the demand.

Fig. 8 shows the results of the energy resource management for this scenario.

\subsection{Scenario 2}

In Scenario 2 the generation of wind farms is low all day long. In periods 10 to 12 the wind generation is zero. The main problem is that the forecasted generation is higher than the actual one, being necessary to use additional resources.

In the context that corresponds to this scenario (context 3), the DNO has contracts that allow the use of two steps of two hydro units and LC_2-1 and LC_2-2. As a result of the optimization process, the DNO uses two steps of $\mathrm{H} 1$ and the first step of $\mathrm{H} 2$ as additional resources to compensate the lack of wind generation.

Fig. 9 shows the results of the energy resource management for scenario 2 .

\subsection{Scenario 3}

Scenario 3 simulates a situation of a double contingency. The first fault occurs in the bus 108 substation. This substation is out of service between periods 16 and 20, and it was scheduled to supply $3 \mathrm{MWh}$ in each of these periods. The second fault occurs in the MSW generator connected to bus 76, between periods 19 and 22 . This generator should produce 1.2 MWh in each of these periods. In periods 19 and 20 both resources are out of service simultaneously. The impact of this double contingency is very significant because it represents the lack of about $15 \%$ of the total generation capacity.

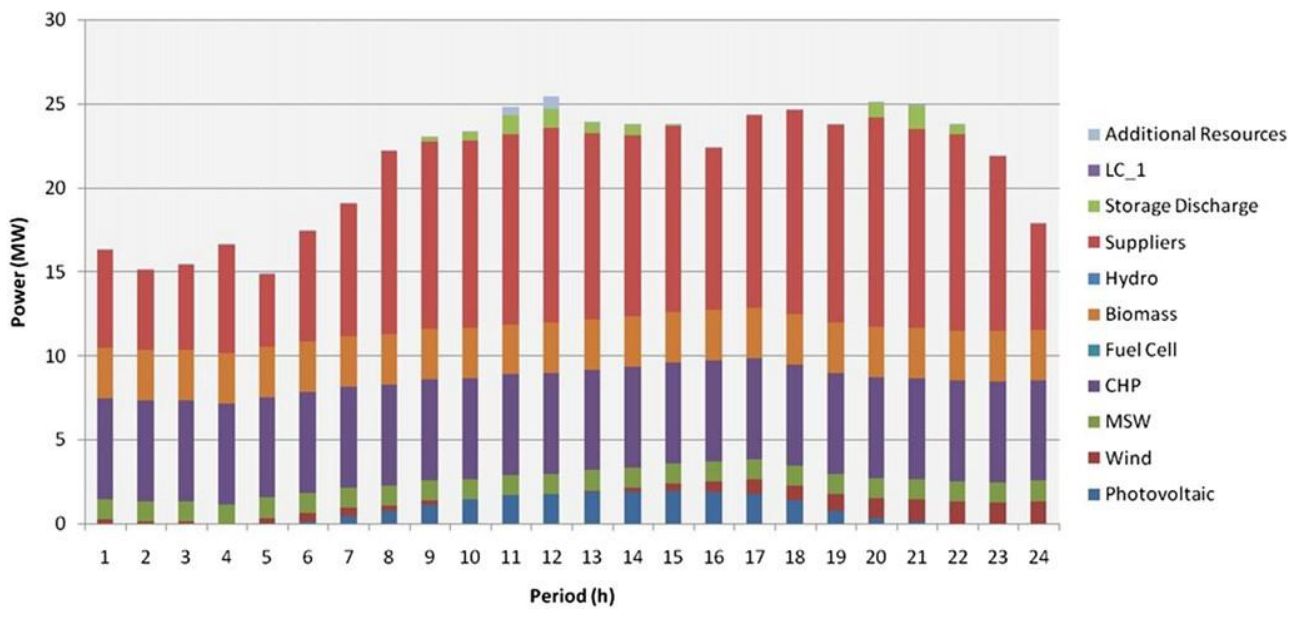

Fig. 9. Energy resource scheduling for scenario 2 . 


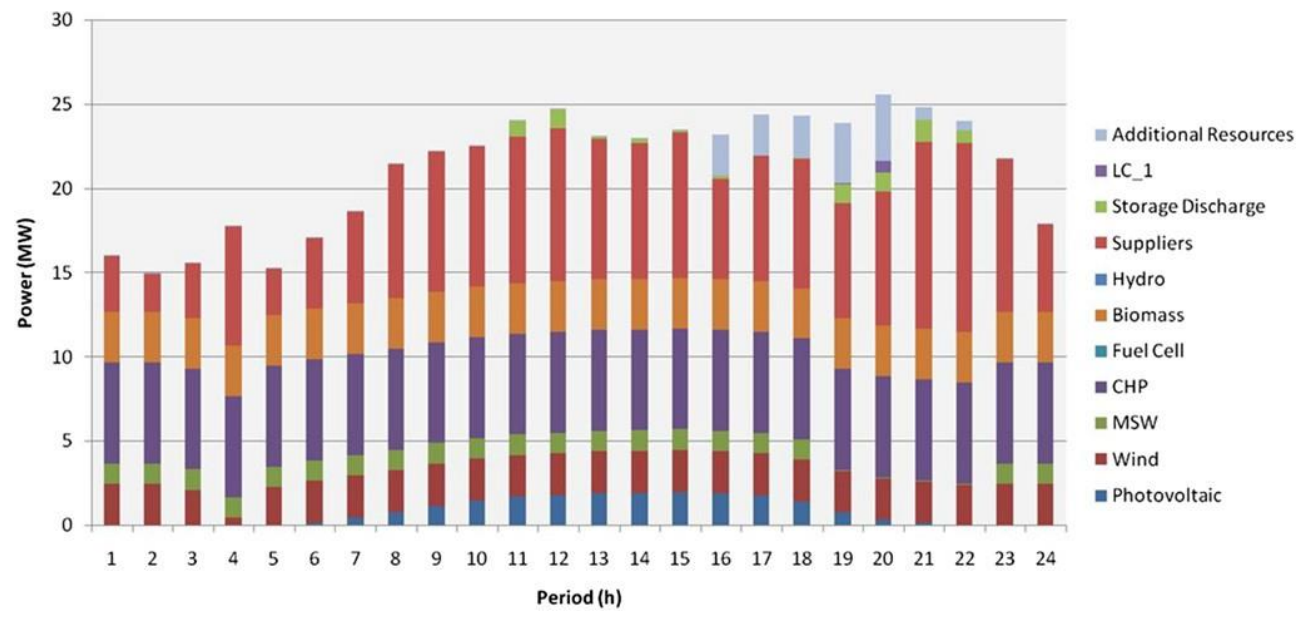

Fig. 10. Energy resource scheduling for scenario 3

In practice we have three different contexts in this scenario. The first context (context 4) occurs between periods 16 and 18, in which the substation is out of service. The second context (context 5) occurs in periods 19 and 20 , in which the substation and generator 76 are out of service. The third context (context 6) occurs in periods 21 and 22 , in which generator 76 is out of service.

During the periods in which the double contingency occurs, it is necessary to use all additional contracted resources and also DLC demand response program corresponding to base resource LC_1.As the resulting LMP value is higher than the LC_2-1 trigger value (3 m.u./kWh), this program is activated. The actual high LMP value is due to the existence of non supplied priority loads.

Fig. 10 shows the results of the energy resource management for scenario 3.

For operational purposes, a transient stability analysis is necessary to assess the system stability state. In the situations for which the system is not stable, the use of ancillary services should be scheduled for the short-term time scale, in order to avoid load shedding.

\section{Conclusions}

In the context of power system operation with intensive use of Distributed Energy Resources (DER) and in a competitive environment, an adequate management of the available energy resources is crucial.

In order to enable efficient operation, SCADA systems should evidence context awareness, allowing each player to access its own and also third party resources contracted for each specific operation context.

This paper has proposed a contextual energy resource management methodology, which is able to model contracted third party resource use. Available resources are dynamically updated according to eventually occurred faults, to the operation context and to the existing contracts.

The application of the proposed model is illustrated with the optimization of a DNO operation costs. The paper includes a case study that considers a 114 bus distribution network. The DNO makes use of its own resources which include Distributed Generation, Storage units, Load Curtailment and external suppliers. It can also use additional contracted resources, according to each operation context, including Real Time Demand Response Programs based on LMP triggered events.

\section{Acknowledgements}

This work is supported by FEDER Funds through COMPETE program and by National Funds through FCT under the projects FCOMP-01-0124-FEDER: PEst-OE/EEI/UI0760/2011, PTDC/EEA-EEL/ 099832/2008, PTDC/SEN-ENR/099844/2008, and PTDC/SEN-ENR/ $122174 / 2010$.

\section{References}

[1] US Department of Energy Electricity Advisory Committee. "Smart grid system report," Re-port by Electricity Advisory Committee; 2009.

[2] Sousa T, Morais H, Vale Z, Faria P, Soares J. "Intelligent energy resource management considering vehicle-to-grid: a simulated annealing approach", submitted for publication on IEEE transaction on smart grid, special issue on transportation electrification and vehicle-to-grid applications; 2010.

[3] Vale Z, Venayagamoorthy K, Ferreira J, Morais H. Computational intelligence applications for future power systems. In: Madureira A, Ferreira J, Vale Z, editors. Computational intelligence for engineering systems, vol. 46 Netherlands: Springer; 2011. p. 176e93.

[4] European technology platform smartgrids e strategic research agenda for Europe's electricity networks of the future. European Commission e Directorate for Research Co-operation Energy; 2007.

[5] Shafiullah G, Amanullah M, Ali A, Jarvis D, Wolfs P. Prospects of renewable energy e a feasibility study in the Australian context. Renewable Energy 2012; 39(1):183e97.

[6] Nyeng P, Ostergaard J. Information and communications systems for control by-price of distributed energy resources and flexible demand. IEEE Trans actions on Smart Grid 2011;2(2):334e41.

[7] Faria P, Vale Z, Soares J, Ferreira J. "Demand response management in power systems using a particle swarm optimization approach". IEEE Intelligent Systems, in press.

[8] Kirschen D. Demand-side view of electricity markets. IEEE Transactions on Power Systems 2003;18(2):520e7.

[9] Vale Z, Morais H, Silva M, Ramos C. "Towards a future SCADA", IEEE Power and Energy Society General Meeting, Calgary, Canada, 26e30 July 2009.

[10] Pinto T, Morais H, Oliveira P, Vale Z, Praça I, Ramos C. A new approach for multi-agent coalition formation and management in the scope of electricity markets. Energy 2011;36(8):5004e15.

[11] SCADA systems for electric power industry e five year market analysis and technology forecast through 2011. ARC Advisory Group; 2007.

[12] Ilic M. Dynamic monitoring and decision systems for enabling sustainable energy services. Proceedings of the IEEE 2011;99(1):58e79.

[13] Metke A, Ekl R. Security technology for smart grid networks. IEEE Transactions on Smart Grid 2010;1(1):99e107.

[14] Vale Z, Pinto T, Praça I, Morais H. MASCEM: electricity markets simulation with strategic agents. IEEE Intelligent Systems 2011;26(2):9e17.

[15] Yanling Y, Zuyi L, Kui R. Modeling load redistribution attacks in power systems. IEEE Transactions on Smart Grid 2011;2(2):382e90.

[16] Pedrasa M, Spooner T, MacGill I. Coordinated scheduling of residential distributed energy resources to optimize smart home energy services. IEEE Transactions on Smart Grid 2010;1(2):134e43. 
[17] Tongdan J, Mechehoul M. Ordering electricity via internet and its potentials for smart grid systems. IEEE Transactions on Smart Grid 2010; 1(3):302e10.

[18] Roy A, Das S, Basu K. A predictive framework for locationeaware resource management in smart homes. IEEE Transactions on Mobile Computing 2007; 6(11): $1270 \mathrm{e} 83$.

[19] Morais H, Kádár P, Faria P, Vale Z, Khodr H. Optimal scheduling of a renewable micro-grid in an isolated load area using mixed-integer linear programming. Renewable Energy 2010;35(1):151e6.

[20] Menegaki A. A social marketing mix for renewable energy in Europe based on consumer stated preference surveys. Renewable Energy 2012;39(1):30e9.
[21] GAMS-the solver manuals. Washington DC, USA: GAMS Development Corporation; 2007.

[22] IEEE 123 Node Test FeederPower system analysis, computing and economics committee, distribution system analysis subcommittee. IEEE Power Engineering Society; 2004.

[23] Morais H, Ramos S, Vale Z. "MV producers and consumers agents characterization with DSM techniques", 2009 IEEE Bucharest Power Tech Conference, June 28th e July 2nd, Bucharest, Romania; 2009.

[24] ISO New England. ISO New England manuals. available online: http:// www.iso-ne.com/rules_proceds/isone_mnls/index.html. accessed June 2011. 